\title{
Novos registros de moscas ectoparasitas (Diptera, Streblidae e Nycteribiidae) em morcegos (Mammalia, Chiroptera) do Amazonas e Pará, Brasil ${ }^{1}$
}

\author{
Gustavo Graciolli ${ }^{2}$ \\ Enrico Bernard $^{3}$
}

\begin{abstract}
New records of batflies (Diptera, Streblidae and Nycteribiidae) on bats (Mammalia, Chiroptera) from Amazonas and Pará States, Brazil. Species of batflies of bats from Amazonas and Pará, Brazil are reported. Eleven species of Streblidae and three of Nycteribiidae were found on nine species of bats, belonging to seven genera. Pseudostrebla greenwelli Wenzel, 1996, Trichobius affinis Wenzel, 1976, Trichobius silvicolae Wenzel, 1976, and Hershkovitzia inaequalis Theodor, 1967 were collected for the first time in Brazil. Strebla consocia Wenzel, 1966, Strebla galindoi Wenzel, 1966, Trichobius dugesioides phyllostomus Guerrero, 1998, and Trichobius joblingi Wenzel, 1966 are new records for state of Amazonas. Noctiliostrebla maai Wenzel, 1966, Basilia dubia Guimarães \& D'Andretta, 1956, and Basilia ferruginea Miranda Ribeiro, 1903 are new records for state of Pará.

KEY WORDS. Nycteribiidae, Streblidae, batflies, bats, Amazônia
\end{abstract}

Cerca de 6.000 espécies de insetos pertencentes a sete ordens são conhecidas como ectoparasitos de vertebrados de sangue quente (MARSHALL 1982), das quais cerca de 700 parasitam morcegos. A relação hospedeiro-parasito envolvendo morcegos é bastante estreita, existindo famílias de dípteros como Nycteribiidae e Streblidae, que são exclusivamente encontradas em associação com morcegos (WENZEL 1970).

Na América do Sul, informações sobre a distribuição de parasitos e espécies de morcegos hospedeiras são conhecidas para: Venezuela (WENZEL 1976; GUERRERO 1993; 1994a,b; 1995a,b; 1996a), Colômbia (MARINKELLE \& GROSE 1981), Peru (GUERRERo 1996b) e Argentina (AUTINO et al. 1999). Na Região Norte do Brasil foram registradas 37 espécies pertencentes a 13 gêneros de Streblidae (GuimarÃes 1937; Jobling 1939, 1949; WenZel et al. 1966; WeNZEL 1970; GUERRERO 1994a,b, 1995a,b, 1996a, 1997; GRACIOLli \& LiNARDI, no prelo) e quatro espécies de dois gêneros de Nycteribiidae (GUIMARÃES \& D'ANDRETTA 1956; PETESON \& LACEY 1985; GRACIOLli \& LinARDI, no prelo). Ainda que a Região Norte seja composta por sete estados, apenas três desses dispõem de

1) Publicacão número 364 da série técnica do PDBFF. Contribuição número 1339 do Departamento de Zoologia, Universidade Federal do Paraná.

2) Departamento de Zoologia, Universidade Federal do Paraná. Caixa Postal 19020, 81531-980 Curitiba, Paraná, Brasil. E-mail: mingau@ bio.ufpr.br

3) Department of Biology, York University. 4700 Keele St., Toronto, Ontario Canada M3J 1P3. E-mail: ebernard@yorku.ca 
informações sobre ectoparasitas de morcegos: Amazonas com quatro espécies de Streblidae e duas de Nycteribiidae, Pará com 26 de Streblidae e Rondônia com 13 de Streblidae. As moscas estréblidas foram coletadas sobre morcegos das famílias Noctilionidae e Phyllostomidae e as nicteribidas sobre Vespertilionidae e Thyropteridae.

São apresentados aqui os resultados de uma pequena amostragem de dípteros ectoparasitos de morcegos, capturados em duas áreas no Amazonas (Rio Preto da Eva) e Pará (Alter do Chão). São apresentados também comentários sobre os táxons encontrados, seus hospedeiros e distribuição geográfica.

\section{MATERIAL E MÉTODOS}

Os ectoparasitos foram coletados durante dois estudos independentes: efeitos da fragmentação florestal sobre comunidades de morcegos e uso de florestas, fragmentos florestais e savanas por morcegos.

O primeiro estudo se estendeu de janeiro de 1996 a julho de 1999 (SAMPAIO et al., no prelo), mas as coletas dos ectoparasitos aqui relacionados se restringiram aos meses de março e maio de 1999. Os morcegos foram capturados nas reservas do Projeto Dinâmica Biológica de Fragmentos Florestais (PDBFF), um convênio entre o Instituto Nacional de Pesquisas da Amazônia (INPA) e a Smithsonian Institution dos Estados Unidos. As reservas do PDBFF ( $2^{\circ} 24^{\prime} \mathrm{S}, 59^{\circ} 43^{\prime} \mathrm{W}$ e $2^{\circ} 25^{\prime} \mathrm{S}$, $59^{\circ} 45^{\prime} \mathrm{W}$ ) localizam-se no município de Rio Preto da Eva, a cerca de $90 \mathrm{~km}$ de Manaus, em uma área que contém fragmentos florestais de tamanhos variados e remanescentes de floresta primária (BIERREGAARD 1990). Na região das reservas do PDBFF foram registradas até o momento 77 espécies de morcegos, pertencentes a sete famílias (SAMPAIO et al., no prelo).

O segundo estudo foi realizado entre abril e dezembro de 2000 , junto a vila de Alter do Chão ( $2^{\circ} 30^{\prime} \mathrm{S}, 5^{\circ} 57^{\prime} \mathrm{W}$ ), cerca de $40 \mathrm{~km}$ distante da cidade de Santarém, Pará, na margem direita do Rio Tapajós. Alter do Chão está em uma área relativamente seca, onde predominam florestas tropicais e savanas amazônicas (PIRES \& PRANCE 1985; SANAIOTTI \& MAGNUSSON 1995). A vegetação de savana é estruturalmente similar ao cerrado do Brasil Central, porém floristicamente diferente, sendo composta por gramíneas e aglomerações esparsas de arbustos, de espécies das famílias Myrtaceae e Melastomataceae (MIRANDA 1993). Fragmentos florestais variando de 0,2 a 360 ha também ocorrem nas áreas de savana. Até o momento foram registrados em Alter do Chão 72 espécies de morcegos, pertencentes a 40 gêneros e sete famílias (BERNARD 2001; E. Bernard, dados não publicados). Entretanto, indicadores de riqueza de espécies mostram que esse número pode chegar a mais de 90 espécies, caracterizando Alter do Chão como uma das localidade com maior diversidade de espécies de morcegos da América Latina (SIMMONS \& VOSS 1998; LIM \& ENGSTROM 2001). As savanas amazônicas têm uma distribuição restrita e as áreas ao redor de Alter do Chão foram sugeridas para designação como Área de Proteção Ambiental (A. Albernaz, com. pess.) e santuário de fauna (RYLANDS \& PINTO 1998).

A metodologia utilizada para a captura dos morcegos foi similar em ambos os estudos, com a utilização de redes de captura armadas entre 0 e 2,5 m do nível

Revta bras. Zool. 19 (Supl. 1): $77-86,2002$ 
do solo, tanto em áreas de mata primária quanto nos fragmentos (SAMPAIO et al., no prelo; BERNARD 2001). Os indivíduos capturados foram medidos, sexados e identificados conforme uma chave de identificação para morcegos da Amazônia (C.O. Handley, com. pess.).

Foram examinados morcegos pertencentes a nove espécies e sete gêneros (Tab. I). O critério de seleção das espécies de morcegos foi a raridade (Thyroptera lavali, Lasiurus cf. blossevillii e Myotis sp.) ou a presença evidente dos ectoparasitos (demais espécies). Os morcegos foram examinados com o deslocamento do pêlo e parte dos ectoparasitos foi coletada com o auxílio de pinça. Ectoparasitos coletados em um mesmo morcego foram agrupados em um recipiente contendo as informações específicas daquele hospedeiro. Espécies de Nycteribiidae e Streblidae foram identificadas segundo GUIMARÃES \& D'ANDRETTA (1956), PETERSON \& LACEY (1985) e GUERRERO (1993 a 1996a). Os ectoparasitos coletados foram depositados no Setor de Coleções Entomológicas do INPA e na Coleção de Entomologia Pe. J.S. Moure, Departamento de Zoologia, Universidade Federal do Paraná, Curitiba, Paraná.

\section{RESULTADOS E DISCUSSÃO}

Um total de 54 ectoparasitos ( 27 machos e 27 fêmeas) foram coletados sobre nove espécies de morcegos (Tab. I). Treze morcegos (cinco machos e oito fêmeas) foram analisados e 11 espécies de sete gêneros de Streblidae e três espécies de dois gêneros de Nycteribiidae foram encontradas (Tab. I).

Informações referentes aos hospedeiros e ectoparasitos coletados e comentários sobre a distribuição e ocorrência das espécies são apresentadas a seguir. Para os hospedeiros, os valores entre parênteses referem-se ao peso em gramas e comprimento do antebraço (ab) em mm.

\section{Noctilionidae}

\section{Noctilio albiventris Desmarest, 1818}

Foram examinados dois machos adultos $(27 \mathrm{~g} ., \mathrm{ab}=56 \mathrm{~mm} ; 29 \mathrm{~g}$., $\mathrm{ab}=58$ $\mathrm{mm})$ e três fêmas adultas ( $25 \mathrm{~g}$., $\mathrm{ab}=56 \mathrm{~mm} ; 29 \mathrm{~g}$., $\mathrm{ab}=57 \mathrm{~mm} ; 27 \mathrm{~g} ., \mathrm{ab}=61$ $\mathrm{mm}$ ), capturados em 31/VII/2000, quando deixavam um abrigo em Alter do Chão. Noctilio albiventris é uma espécie amplamente distribuída, do sul do México ao norte da Argentina (NOWAK 1994). Esta espécie se alimenta de insetos, capturados na superfície da água, e geralmente se abriga em ocos de árvores, na folhagem das árvores ou em construções humanas. Possuem pelagem extremamente curta, com a coloração variando consideravelmente entre indivíduos e localidades, desde de amarelado até castanho. Podem formar colônias com até 600 indivíduos (HOOD \& PITOCCHELli 1983). Em Alter do Chão, os morcegos ocupavam o telhado de uma casa, em um grupo de cerca de 50-60 indivíduos. Alguns destes estavam intensamente parasitados, principalmente por carrapatos do gênero Ornithodoros Koch, 1844.

Ectoparasitos. Noctiliostrebla maai Wenzel, 1966 (Diptera, Streblidae): quatro machos e duas fêmeas. Novo registro para o Pará. GUERRERo (1995b) registrou-a para o Rondônia sobre Noctilio albiventris. Noctiliostrebla maai é a 
única espécie do gênero que parasita exclusivamente $N$. albiventris. Em levantamento realizado na Venezuela, WENZEL (1976) encontrou 99\% dos indivíduos de $N$. maai sobre $N$. albiventris.

Paradyschiria parvula Wenzel, 1966 (Diptera, Streblidae): quatro machos e cinco fêmeas. Esta espécie foi registrada no Mato Grosso (GUERRERo 1995b) e Pará (WENZEL 1970) sem dados sobre o hospedeiro e Rondônia sobre N. albiventris (GUERRERO 1995b). Na Venezuela, WENZEL (1976) coletou 99,5\% dos exemplares desta espécie coletados sobre $N$. albiventris.

Tabela I. Ectoparasitos coletados em nove espécies de morcegos em Rio Preto da Eva (Amazonas) e Alter do Chão (Pará), com indicações de novos registros para os estados ou território nacional.

\begin{tabular}{|c|c|c|c|c|c|c|}
\hline \multirow{2}{*}{ Hospedeiros } & \multicolumn{2}{|c|}{$\mathrm{N}$ (hospedeiro) } & \multirow{2}{*}{ Ectoparasitos } & \multicolumn{2}{|c|}{ N (ectoparasita) } & \multirow{2}{*}{$\begin{array}{l}\text { Primeiro } \\
\text { registro para }\end{array}$} \\
\hline & Machos & Fêmeas & & Machos & Fêmeas & \\
\hline \multirow[t]{2}{*}{ Noctilio albiventris } & 2 & 3 & Noctiliostrebla maai & 4 & 2 & Pará \\
\hline & & & Paradyschiria parvula & 4 & 5 & \\
\hline \multirow[t]{3}{*}{ Phyllostomus elongatus } & - & 1 & Trichobius joblingi & 2 & 1 & Amazonas \\
\hline & & & T. dugesioides phyllostomus & 2 & - & Amazonas \\
\hline & & & Strebla consocia & 2 & 2 & Amazonas \\
\hline \multirow[t]{3}{*}{ Tonatia brasiliense } & - & 1 & Trichobius affinis & - & 1 & Brasil \\
\hline & & & Pseudostrebla greenwelli & 1 & 1 & Brasil \\
\hline & & & Mastoptera minuta & 4 & 4 & \\
\hline Tonatia silvicola & - & 1 & Trichobius silvicolae & 3 & 2 & Brasil \\
\hline Tonatia saurophila & 1 & 1 & Strebla galindoi & 1 & 1 & $\begin{array}{l}\text { Amazonas e para } \\
\text { a espécie } \\
\text { hospedeira }\end{array}$ \\
\hline Artibeus jamaicensis & - & 1 & Megistopoda aranea & 1 & 1 & \\
\hline Lasiurus cf. blossevilli & 1 & - & Basilia ferruginea & - & 2 & Pará \\
\hline Myotis sp. & 1 & - & Basilia dubia & 2 & 5 & $\begin{array}{l}\text { Pará e para a } \\
\text { espécie } \\
\text { hospedeira }\end{array}$ \\
\hline Thyroptera lavali & - & 1 & Hershkovitzia inaequalis & 1 & - & Brasil \\
\hline Total & 5 & 8 & & 27 & 27 & \\
\hline
\end{tabular}

\section{Phyllostomidae}

\section{Phyllostomus elongatus (E. Geoffroy, 1810)}

Uma fêmea adulta (41 g.), capturada em 12/V/1999 em região de mata primária nas reservas do PDBFF. Phyllostomus elongatus tem uma distribuição restrita à América do Sul, ao leste dos Andes, da Colômbia a Bolívia e sudoeste do Brasil (NowAK 1994). É uma espécie predominante insetívora, e está geralmente associada à áreas de mata. Abriga-se em ocos de árvores, formando colônias de sete a 15 indivíduos (TUTTLE 1970), onde podem ser encontrados simpatricamente com outras espécies de morcegos (SIMMONS \& Voss 1998). Possuem pelagem relativamente longa e densa, geralmente de coloração castanho claro a escuro.

Ectoparasitos. Trichobius dugesioides phyllostomus Guerrero, 1998 (Diptera, Streblidae): dois machos. Espécie pertencente ao complexo parasiticus (WENZEL et al. 1966). Primeiro registro desta subespécie no território brasileiro, estando anteriormente restrita à Venezuela. Até o momento, esta subespécie de Trichobius dugesioides é encontrada somente sobre P. elongatus (GUERRERO 1998).

Revta bras. Zool. 19 (Supl. 1): 77 - 86, 2002 
Trichobius joblingi Wenzel, 1966 (Diptera, Streblidae): dois machos e uma fêmea. Espécie pertencente ao complexo dugesii (WENZEL et al. 1966). Novo registro para o Amazonas. GUERRERO (1995a, 1997) registrou-a sobre Carollia perspicillata (Linnaeus, 1758) em Rondônia e sobre Artibeus anderseni Osgood, 1916 e Platyrrhinus helleri (Peters, 1866) em Belém, Pará. Embora seja um parasito primário de $C$. perspicillata, $T$. joblingi é encontrada sobre um grande número de espécies de morcegos (GUERRERO 1995a).

Strebla consocia Wenzel, 1966 (Diptera, Streblidae): dois machos e duas fêmeas. Novo registro para o Amazonas. Restrita à América do Sul (GUERRERO 1996a), foi registrada por GUERRERO (1997) no município de Belém, Pará, sobre Phyllostomus hastatus (Pallas, 1767).

\section{Tonatia brasiliense (Peters, 1866)}

Fêmea grávida (12 g., ab $=34 \mathrm{~mm})$, capturada em 22/VIII/2000, em rede armada na savana em Alter do Chão. Tonatia brasiliense possui uma distribuição ampla, do sul do México até o nordeste da Bolívia e leste do Brasil, e também em Trinidad (NOWAK 1994). Utiliza áreas de floresta e pode se abrigar em cupinzeiros abandonados. É uma espécie predominantemente insetívora, mas frutos podem fazer parte de sua dieta. Possuem pelagem de comprimento médio e densa, com coloração variando entre cinza e castanho.

Ectoparasitos. Trichobius affinis Wenzel, 1976 (Diptera, Streblidae): uma fêmea. Novo registro para o Brasil. Espécie pertencente ao grupo longipes (WENZEL et al. 1966). Anteriormente, esta espécie havia sido encontrada apenas na Venezuela sobre Tonatia brasiliense (GUERRERO 1994a).

Pseudostrebla greenwelli Wenzel, 1966 (Diptera, Streblidae): um macho e uma fêmea. Novo registro para o Brasil. Espécie muito rara em coleções. São conhecidos apenas quatro exemplares coletados no Panamá e Venezuela (GUERRERO 1994b).

Mastoptera minuta (Costa Lima, 1921) (Diptera, Streblidae): quatro machos e quatro fêmeas. Espécie registrada sobre Phyllostomus hastatus no Pará (JoBLING 1949) e Rondônia (GUERRERO 1995b). WENZEL (1976) considerou apenas os indivíduos encontrados sobre Tonatia silvicola (D’Orbigny, 1836) como M. minuta. Os indivíduos coletados sobre outros hospedeiros (espécies de Tonatia Gray, 1827 e Phyllostomus Lacépède, 1799) foram considerados pertencentes ao complexo "minuta".

\section{Tonatia silvicola (D’Orbigny, 1836)}

Uma fêmea adulta (37 g.) capturada em 12/V/1999, em região de mata primária no PDBFF. Espécie de distribuição tropical, presente em zonas de vegetação variada (de floresta à áreas secas) da Nicarágua ao Brasil (exceto sul), Bolívia e Paraguai (NOVAK 1994). Utiliza como abrigo cupinzeiros abandonados, formando pequenos grupos familiares de seis a 16 indivíduos. Possuem pelagem densa e longa, variando entre cinza claro até castanho escuro. Esta espécie já foi encontrada em associação com 41 outras espécies de morcegos. Se alimenta predominantemente de insetos, mas pode ingerir também frutos, existindo ainda relatos da presença de ossos e carne em seus estômagos (MEDELLIN \& ARITA 1989). 
Ectoparasitos. Trichobius silvicolae Wenzel, 1976 (Diptera, Streblidae): três machos e duas fêmeas. Espécie pertencente ao grupo longipes (WENZEL et al. 1966). Primeiro registro em território brasileiro, estando esta espécie anteriormente restrita à Venezuela, onde parasita várias espécies de Tonatia (GUERRERO 1994a).

\section{Tonatia saurophila Koopman \& Willians, 1951}

Um macho adulto (30 g.), capturado em 04/IV/1999 em região de mata primária no PDBFF. Esta espécie distribui-se do sul do México e Belize, até a porção sul do Peru e leste do Brasil (NowaK 1994). Existe sobreposição de habitats e de dieta entre $T$. saurophila e $T$. silvicola, e de maneira geral a biologia e ecologia desta espécie se aproxima bastante de $T$. silvicola. A coloração do pêlo varia de cinza claro a castanho.

Ectoparasitos. Strebla galindoi Wenzel, 1966 (Diptera, Streblidae): um macho e uma fêmea. Novo registro para o Amazonas. Primeiro registro de parasitismo sobre $T$. saurophila por Streblidae. Esta espécie já havia sido registrada em Belém por GUERRERO (1997), sobre Tonatia bidens (Spix, 1823).

\section{Artibeus jamaicensis Leach, 1821}

Fêmea adulta lactante $(50 \mathrm{~g} ., \mathrm{ab}=60 \mathrm{~mm})$, capturada em 7/V/2000, em rede armada na savana em Alter do Chão. Artibeus jamaicensis apresenta uma das mais amplas distribuições entre as espécies de morcegos neotropicais, desde a região central do México até o sul do Brasil, incluindo várias ilhas do Caribe, e ocupando diversos tipos de habitats (ORTEGA \& CASTRO-ARELLANO 2001). Esta é também uma das espécies mais estudadas entre o quirópteros, e tem sido registrada abrigando-se em cavernas, ocos de árvores, edificações ou em contato com a folhagem, formando grupos sociais com poucos indivíduos. O comprimento do pêlo é médio e de textura macia, variando do cinza claro ao castanho claro. Artibeus jamaicensis é um frugívoro generalista e sua dieta pode incluir dezenas de espécies de plantas, alimentando-se de frutos inteiros, casca, polpa, flores, pólen ou néctar, e ainda folhas (ORTEGA \& CASTRO-ARELLANO 2001). Insetos em pequenas quantidades também já foram registrados na dieta desta espécie.

Ectoparasitos. Megistopoda aranea (Coquillett, 1899) (Diptera, Streblidae): um macho e uma fêmea. Previamente, $M$. aranea havia sido encontrada em Rondônia sobre Artibeus lituratus (Olfers, 1818) (GUERRERO 1994b) e no Pará (WENZEL 1970). Esta espécie tem como principal hospedeiro no Panamá e norte da América do Sul, Artibeus jamaicensis. Em levantamentos realizados respectivamente no Panamá e Venezuela, 54,4\% (WENZEL et al. 1966) e 97\% (WENZEL 1976) de $M$. aranea foram coletados sobre $A$. jamaicensis.

\section{Vespertilionidae}

\section{Lasiurus cf. blossevillii (Lesson \& Carmot, 1826)}

Macho adulto $(8 \mathrm{~g} ., \mathrm{ab}=39 \mathrm{~mm})$, capturado em 01/IX/2000 em rede armada na savana em Alter do Chão. Esta espécie apresenta uma ampla distribuição, desde o sudoeste dos Estados Unidos até a Argentina (EISENBERG \& REDFORD 1999). Lasiurus blossevillii é uma espécie pouco conhecida, mas as espécies deste gênero

Revta bras. Zool. 19 (Supl. 1): 77 - 86, 2002 
são exclusivamente insetívoras e abrigam-se em contato com a folhagem e eventualmente no oco de árvores ou edificações. Algumas espécies são migratórias, deixando as áreas mais frias durante o inverno (NOWAK 1994). Apresentam hábitos solitários, mas as fêmeas podem formar pequenas colônias durante a maternidade. Lasiurus blossevillii pertence ao grupo borealis, cuja pelagem é longa, densa e macia e caracterizada pela predominância da coloração avermelhada no dorso e rajada de preto, amarelo e branco no ventre.

Ectoparasitos. Basilia ferruginea Miranda Ribeiro, 1903 (Diptera, Nycteribiidae): duas fêmeas. Novo registro para o Pará. Espécie pertencente ao grupo ferruginea (THEODOR 1967). Caso seja confirmada a identificação do hospedeiro, trata-se de um novo registro de hospedeiro para $B$. ferruginea. Anteriormente, esta mosca nicteribida havia sido encontrado em Lasiurus borealis (Müller, 1776) (GUIMARÃES \& D'ANDRETTA 1956; GUIMARÃES 1966; WhITAKER \& MCCRACKEN 2001) e Lasiurus cinereus (Beauvois, 1796) (WHITAKER \& MCCRACKEN 2001).

\section{Myotis sp.}

Um macho adulto, capturado em Alter do Chão. Os indivíduos examinados referem-se a uma nova espécie do gênero Myotis, que se encontra em processo de descrição (E. Bernard, dados não publicados). Tal espécie se assemelha a Myotis albescens (E. Geoffroy, 1806) no tamanho, entretanto difere desta em características do crânio e principalmente na coloração das asas e uropatágio. A pelagem é curta, densa e macia, bicolor (cinza e castanho). Espécies do gênero Myotis são exclusivamente insetívoras e pouco se sabe sobre os abrigos utilizados pelas espécies presentes na Amazônia.

Ectoparasitos. Basilia dubia Guimarães \& D'Andretta, 1956 (Diptera, Nycteribiidae): dois machos e cinco fêmeas. Novo registro para o Pará e novo registro de hospedeiro. Espécie pertencente ao grupo speiseri (THEODOR 1967). Registrada no Amazonas por GUIMARÃES \& D'ANDRETTA (1956) parasitando Myotis nigricans (Schinz, 1821). GUIMARÃES $(1972,1977)$ registrou B. dubia sobre M. albescens na Venezuela.

\section{Thyropteridae}

\section{Thyroptera lavali Pine, 1993}

Uma fêmea adulta (6 g) não lactante capturada em 09/V/2000 em uma área de mata em Alter do Chão. Este exemplar representa a primeira ocorrência desta espécie de morcego em território brasileiro (E. Bernard, dados não publicados). Thyroptera lavali é uma espécies pouco conhecida, descrita a partir de indivíduos coletados em uma localidade no norte do Peru (PINE 1993), a cerca de $2100 \mathrm{~km}$ a noroeste de Alter do Chão, e representada ainda por três indivídủos coletados no Parque Nacional Yasuni, na Província de Napo, Equador (ReID et al. 2000). As espécies deste gênero são insetívoras e tem como peculiaridade o fato de utilizarem os discos adesivos presentes nas asas e pés para se fixarem em contato direto com a folhagem. Apresenta pelagem curta, grossa e esparsa, de coloração castanho escura. 
Ectoparasitos. Hershkovitzia inaequalis Theodor, 1967 (Diptera, Nycteribiidae): um macho. Novo registro para o Brasil. As espécies de Hershkovitzia Guimarães \& D'Andretta, 1956, gênero endêmico do continente americano, parasitam morcegos do gênero Thyroptera Spix, 1823. Hershkovitzia primitiva Guimarães \& D'Andretta, 1956 e H. inaequalis são encontradas sobre Thyroptera discifera (Lichtenstein \& Peters, 1855), Hershkovitzia cabala Peterson \& Lacey, 1985 sobre Thyroptera tricolor Spix, 1823 e não há informações sobre o hospedeiro de Hershkovitzia coeca Theodor, 1967. Hershkovitzia inaequalis foi descrita a partir de seis espécimens (um macho e cinco fêmeas) que estão depositados no Field Museum of Natural History (FMNH), Chicago. Os dados de procedência destes exemplares estão citados da seguinte forma em THEODOR (1967: 352): “...PERU. Loreto, from Thyroptera discifera, 16.ix.1957, Maynas, Celestino, Kalinowsky, + holotype, $1 \sigma^{x} 4$ 우 paratypes. Chicago Natural History Museum.". No trabalho de PINE (1993: 214) no qual foi descrita T. lavali de material depositado no FMNH, nos dados de procedência do holótipo há a seguinte informação: “...Quebrada. Esperanza, Río Javary-Mirim, Loreto, Peru. Altitude 200 meters" according BLAKE (1959); taken on 16 September 1957 by C[elestino]. Kalinowski, original filed no.=2452...". Assim como no material examinado por PINE (1993) não há exemplares de T. discifera com dados semelhantes ao holótipo da espécie descrita, é plausível considerarmos que o hospedeiro-tipo de $H$. inaequalis seja $T$. lavali e não $T$. discifera.

O presente estudo sobre ectoparasitos de morcegos se restringiu a um pequeno número de indivíduos e de espécies de morcegos, o que não nos permite examinar com maior profundidade a relação parasito/hospedeiro entre as espécies registradas. Mesmo assim, quatro novos registros foram acrescentados para o território brasileiro, além da expansão da área de distribuição para várias das espécies de ectoparasitos encontradas. É muito provável que uma parcela significativa da diversidade de espécies de ectoparasitos de morcegos presentes na região Amazônica ainda permaneça desconhecida pela ciência, bem como a extensão e refinamento de suas relações com as espécies hospedeiras. A grande diversidade de espécies e a escassez de estudos específicos com morcegos reforçam esta hipótese e os resultados aqui apresentados ressaltam a extrema necessidade de um trabalho aprofundado para a região Amazônica, envolvendo a identificação, biologia e ecologia de ectoparasitos de morcegos.

AGRADECIMENTOS. Agradecemos ao Instituto Nacional de Pesquisas da Amazônia (INPA) e ao Projeto Dinâmica Biológica de Fragmentos Florestais (PDBFF), pela utilização da infraestrutura das reservas em Manaus. A captura dos morcegos em Manaus foi possível graças ao apoio financeiro provenientes dos Drs. Charles Handley, Elisabeth Kalko e Érica Sampaio, e apoio logístico de Francisco Marques, José Tenasol e Jerônimo Andes. As atividades em Alter do Chão foram possíveis através da utilização da infraestrutura oferecida pelo INPA, através do Dr. William Magnusson, e de bolsas da CAPES e CNPq (doutorado) e York University para o segundo autor. Edivaldo Farias, Adnor Farias Costa e Caroline Aires auxiliaram na captura dos morcegos e coleta dos parasitos. 


\section{REFERÊNCIAS BIBLIOGRÁFICAS}

AUtINO, A.G.; G.L. ClAPS \& R.M. BARQUEZ. 1999. Insectos ectoparasitos de murcielagos de las Yungas de la Argentina. Acta Zool. Mex., n. s., 78: 119-169.

Bernard, E. 2001. Species list of bats (Mammalia, Chiroptera) in Santarém area, Pará State, Brazil. Revta bras. Zool. 18: 455-463.

BierregaARD, R.O. 1990. Central Amazonian forests and the Minimal Critical Size of Ecosystems Project, p. 60-75. In: A. Gentry (Ed.). Four Neotropical Rainforest. New Haven, Yale Univ. Press, 627p.

BLAKE, E.R. 1959. Two new game birds from Peru. Fieldiana, Zool., 39: 373-376.

EISENBERG, J.F. \& K.H. ReDFoRD. 1999. Mammals of the Neotropics. The Central Neotropics. Chicago, Univ. Chicago Press, vol. 3, p.117-229.

Guerrero, R. 1993. Catalogo de los Streblidae (Diptera: Pupipara) parasitos de murcielagos (Mammalia: Chiroptera) del Nuevo Mundo. I. Clave para los generos y Nycterophilinae. Acta Biol. Venez 14: 61-75.

1994a. Catalogo de los Streblidae (Diptera: Pupipara) parasitos de murcielagos (Mammalia: Chiroptera) del Nuevo Mundo. II. Los grupos: pallidus, caecus, major, uniformis y longipes del genero Trichobius Gervais, 1844. Acta Biol. Venez. 15: 1-18.

1994b. Catalogo de los Streblidae (Diptera: Pupipara) parasitos de murcielagos (Mammalia: Chiroptera) del Nuevo Mundo. IV. Trichobiinae com alas desarrolladas. Bol. Entomol. Venez., n. s., 9: 161-192.

1995a. Catalogo de los Streblidae (Diptera: Pupipara) parasitos de murcielagos (Mammalia: Chiroptera) del Nuevo Mundo. III. Los grupos: dugesii, dunni y phyllostomae del genero Trichobius Gervais, 1844. Acta Biol. Venez. 15: 1-27.

-1995b. Catalogo de los Streblidae (Diptera: Pupipara) parasitos de murcielagos (Mammalia: Chiroptera) del Nuevo Mundo. V. Trichobiinae con alas reducidas o ausentes y miscelaneos. Bol. Entomol. Venez., Nueva Serie 10: 135-160.

-1996a. Catalogo de los Streblidae (Diptera: Pupipara) parasitos de murcielagos (Mammalia: Chiroptera) del Nuevo Mundo. VI. Streblinae. Acta Biol. Venez. 16: 1-26.

— 1996b. Streblidae (Diptera: Pupipara) parásitos de los murciélagos de Pakitza, Parque Nacional Manu (Perú), p. 627-641. In: D.E. Wilson \& A. SAndoval (Eds). MANU: La biodiversidad del sureste del Perú. Washington, DC, Smithsonian Institution, 679p.

- 1997. Catalogo de los Streblidae (Diptera: Pupipara) parasitos de murcielagos (Mammalia: Chiroptera) del Nuevo Mundo. VII. Lista de especies, hospedadores y paises. Acta Biol. Venez. 17: 9-24.

Guerrero, R. 1998. Notes on Neotropical batflies (Diptera, Streblidae). I. The genus Trichobius, with description of two new species and new subspecies from Venezuela. Acta Parasitol. 43: 86-93.

GuimarãES, L.R. 1937. Sobre as especies sul americanas do genero Trichobius (Diptera-Streblidae). Revta Mus. Paul. 23: 653-666.

- 1966. Nycteribiid batflies from Panama (Diptera: Nycteribiidae), p. 393-404. In: R.L. WenZel \& V.J. TiPton (Eds). Ectoparasites of Panama. Chicago, Field Mus. Nat. Hist., XII+861p. . 1972. Venezuelan nycteribiid batflies (Diptera: Nycteribiidae). Brigham Young Univ. Sci. Bull., Biol. Ser., 17: 1-11.

. 1977. Supplementary note on Venezuelan batflies (Diptera: Nycteribiidae). Gt. Basin Nat. 37: 221-224.

Guimarães, L.R. \& M.A.V. D'ANDRETtA. 1956. Sinopse dos Nycteribiidae (Diptera) do Novo Mundo. Arq. Zool. São Paulo 10: 1-184.

Graciolli, G. \& P.M. LiNARDI (no prelo). Some Streblidae and Nycteribiidae (Diptera: Hippoboscoidea) from Maracá Island, Rorraima, Brazil. Mem. Inst. Oswaldo Cruz.

Hood, C.S. \& J. PrtocCHeLli. 1983. Noctilio albiventris. Mammalian Species 197: 1-5.

Jobling, B. 1939. On some american genera of the Streblidae and their species, with the description of a new species of Trichobius (Diptera, Acalypterae). Parasitology 31: 486-497. 
1949. A revision of the species of the genus Aspidoptera Coquillett, with some notes on the larva and puparium of A. clovisi, and a new synonym. Proc. R. Ent. Soc. Lond. (B) 18: 135-144.

LiM, B.K. \& M.D. Engstrom. 2001. Species diversity of bats (Mammalia: Chiroptera) in Iwokrama Forest, Guyana, and the Guianan subregion: implications for conservation. Biodiv. Conserv. 10: 613-657.

MARINKelle, C.J. \& E.S. Grose. 1981. A list of ectoparasites of Colombian bats. Rev. Biol. Trop. 29: 11-20.

Marshall, A.G. 1982. Ecology of bats, p.369-401.. In: T.H. KunZ (Ed.). Ecology of Bats. New York, Plenum Press, $425 \mathrm{p}$.

MEdellin, R.A. \& H.T. ARITA. 1989. Tonatia evotis and Tonatia silvicola. Mammalian Species 334: $1-5$.

MiRANDA, I.S. 1993. Estrutura do estrato arbóreo do cerrado amazônico em Alter do Chão, Pará, Brasil. Revta Brasil. Bot. 16: 143-150.

NowaK, R.M. 1994. Walker's Bats of the World. Baltimore, The John Hopkins Univ. Press, 287p.

Ortega, J. \& I. Castro-Arellano. 2001. Artibeus jamaicensis. Mammalian Species 662: 1-9.

Peterson, B.V. \& L.A. LACEY. 1985. A new species of Hershkovitzia (Diptera: Nycteribiidae) from Brazil, with a key to the described species of the genus. Proc. Entomol. Soc. Wash. 87: 578-582.

PINE, R.H. 1993. A new species of Thyroptera Spix (Mammalia:Chiroptera: Thyropteridae) from the Amazon Basin of northeastern Perú. Mammalia 57: 213-225.

Pires, J.M. \& G.T. Prance. 1985. The vegetation types of the Brazilian Amazon, p. 109-145. In: G.T Prance \& T.E. Lovejoy (Eds). Key environments: Amazonia. Oxford, Pergamon Press, 442p.

Reid, F.A.; M.D. Engstrom \& B.K. LiM. 2000. Noteworthy records of bats from Ecuador. Acta Chiropterologica 2: 37-51.

RYLANDS, A.B. \& L.P.S. PINTO. 1998. Conservação da Biodiversidade na Amazônia Brasileira: uma análise do sistema de unidades de conservação. Cadernos Fundação Brasileira Desenvolvimento Sustentável 1: 14-15.

Sampaio, E.M.; E.K.V. KalKo; E. Bernard; B.R. Herrera \& C.O. Handley (no prelo). An updated checklist of bats (Chiroptera) in an upland forest in Central Amazon including methodological and conservation considerations. Studies Neotrop. Fauna Environ.

Sanaiotti, T.M. \& W.E. Magnusson. 1995. Effects of annual fires on the production of fleshy fruits eaten by birds in a Brazilian Amazonian savanna. Jour. Trop. Ecol. 11: 53-65.

Simmons, N.B. \& R.S. Voss. 1998. The Mammals of Paracou, French Guiana: a neotropical lowland rainforest fauna. Part 1, Bats. Bull. Amer. Mus. Nat. His. 237: 1-219.

THEODOR, O. 1967. An illustrated catalogue of the Rothschild collection of Nycteribiidae (Diptera) in the British Museum (Natural History) with keys and the short descriptions for the identification of subfamilies, genera, species and subspecies. Brit. Mus. (Nat. His.) Publ. 665: 1-506.

TUTTLE, M.D. 1970. Distribution and zoogeography of Peruvian bats, with commnets on natural history. Univ. Kansas Sci. Bull 49: 45-86.

Wenzel, R.L. 1970. Family Streblidae. In: N. Papavero (Ed). A catalogue of the Diptera of the Americas South of the United States. 100. São Paulo, Museu de Zoologia, 25p.

- 1976. The streblid batflies of Venezuela (Diptera: Streblidae). Brigham Young Univ. Sci. Bull., Biol. Ser. 20: 1-177.

Wenzel, R.L.; V.J. Tipton \& A. KIEWLICZ. 1966. The streblid batflies of Panama (Diptera: Calyptera: Streblidae), p. 405-675. In: R.L. WenZel \& V.J. TIPTON (Eds). Ectoparasites of Panama. Chicago, Field Mus. Nat. Hist., 861p.

WHITAKER JR., J.O. \& G.F. MCCRACKen. 2001. Food and ectoparasites of bats on the Galapagos Islands. Acta Chiropterologica 3: 63-69.

Recebido em 14.IX.2001; aceito em 07.V.2002. 